\title{
Primer registro del género Actinostachys (Schizaeaceae) para ArgentinA
}

\author{
GONZALO J. MARQUEZ1 y HÉCTOR A. KELLER²
}

\begin{abstract}
Summary: First record of the genus Actinostachys (Schizaeaceae) for Argentina. The fern Actinostachys pennula (Schizaeaceae) is cited for the first time from Argentina, on the basis of material recently collected in the northeast of Misiones province. It is provided a description of the species, ecological observations, and illustrations of sporophyte and spores.
\end{abstract}

Key words: Ferns, new record, marsh, Misiones.

Resumen: Se registra por primera vez para la flora Argentina el helecho Actinostachys pennula (Schizaeaceae), sobre la base de material recién recolectado en el nordeste de la provincia de Misiones. Se incluye una descripción de la especie, observaciones ecológicas, e ilustraciones del esporofito y esporas características de la especie.

Palabras clave: Helechos, nuevo registro, bañados, Misiones.

\section{INTRODUCCIÓN}

Los bañados o áreas pantanosas de Misiones, en su mayor parte constituyen sitios de superficie reducida, pero pobremente abordados en los estudios florísticos. Aún con el actual desarrollo de los sistemas de interpretación de imágenes satelitales, en la matriz selvática se presentan como ambientes poco perceptibles, debido a sus escasas dimensiones. Además, muchos de estos humedales son de difícil acceso por encontrarse en el interior de grandes macizos de selva, o bien por las dificultades de acceso que ofrece el suelo anegado y la densa vegetación que suele proliferar en sus sectores abiertos. Estos atributos explican el hecho que en una provincia tan pequeña y tan explorada

\footnotetext{
1 Investigador del CONICET, Cátedra de Palinología, Facultad de Ciencias Naturales y Museo, UNLP, Paseo del Bosque s/n, B1900FWA, La Plata, Argentina. cosme@fcnym.unlp.edu.ar

${ }^{2}$ Investigador del CONICET, Facultad de Ciencias Forestales, UNaM, Instituto de Botánica del Nordeste, (CONICET - UNNE, Facultad de Ciencias Agrarias), C.C. 209, 3400 Corrientes, Argentina. kellerhector@ hotmail.com
}

botánicamente, los bañados selváticos sigan aportando hasta el presente novedades taxonómicas y florísticas, entre ellas nuevas especies para la ciencia (Krapovickas, 2012), un nuevo género (Keller, 2017) e inclusive una nueva subtribu (Keller \& Liede-Schumann, 2017).

Los bañados de Misiones también siguen aportando novedades para el país en lo que respecta a los helechos (Meza Torres et al., 2006; Yañez \& Ponce, 2017). En el contexto de estos hallazgos, en la presente contribución se comunica por primera vez la presencia de Actinostachys pennula (Sw.) Hook como especie integrante de la flora palustre de helechos de Argentina.

Los esporangios con anillo apical constituyen un carácter distintivo del orden Schizaeales Schimp. (Smith et al., 2006; Shen et al., 2018). Los representantes documentados para la flora Argentina que cuentan con dicho atributo se limitan a los géneros Anemia Sw. y Lygodium Sw., los cuales fueron recientemente segregados de la familia Schizaeaceae para formar parte de las familias Anemiaceae Link y Lygodiaceae M.Roem., respectivamente (PPG I, 2016). Por lo tanto, el presente hallazgo permite documentar el primer representante de la familia Schizaeaceae (sensu PPG I, 2016) para Argentina. 


\section{Materiales y Métodos}

El material utilizado en este trabajo fue coleccionado en un viaje de campo realizado en el Departamento de Iguazú (Misiones, Argentina) y fue depositado en el herbario CTES. Los ejemplares fueron herborizados utilizando hojas de papel de diario y alcohol 96 con el fin de ayudar en la deshidratación.

Las esporas, sin tratamiento previo, fueron observadas con el microscopio electrónico de barrido (MEB) JEOL JSMT-100 de la Facultad de Ciencias Naturales y Museo de la UNLP.

Para la determinación del material se utilizaron los trabajos de Bierhorst (1971), da Silva et al. (2015), Prado (2005), Ramos Giacosa \& Barakat (2018), Tryon \& Lugardon (1991) y Wagner \& Quevedo (1985).

\section{Resultados}

Actinostachys pennula (Sw.) Hook. Gen. Fil. t. 111A. 1842. Schizaea pennula Sw. Syn. Fil. 150, 379. 1806. Tipo: America meridionalis, habitat in america calidiore. Anónimo s.n. (Holotypus S!). Figs. 2 y 3.

Terrestre. Rizomas cortos, tuberosos, con pelos castaño-rojizos. Frondes dimorfas, lineares, de 10$45 \mathrm{~cm}$ de altura. Pecíolos cortos, de 1-2 $\mathrm{cm}$ de largo y 1-2 mm de diámetro. Láminas simples, lineares, de 1-3 mm de ancho, glabras, triangulares en corte transversal y con una hilera de estomas a cada lado de la vena media en la cara abaxial; frondes estériles con ápice agudo; frondes fértiles terminando en esporangióforos digitados. Esporangióforos castañooscuros a la madurez, divididos en 5-10 segmentos de $1-5 \mathrm{~cm}$ de largo y margen entero; con paráfisis en la cara abaxial y glabros en la cara adaxial. Esporangios abundantes en 2-4 hileras a cada lado de la vena media en la cara abaxial. Esporas monoletes, con lesuras elevadas y cortas, diámetro ecuatorial mayor $51,5(59,8) 63 \mu \mathrm{m}$ y diámetro polar 29,9 $(33,2) 40,7 \mu \mathrm{m}$, superficie foveloada con esferoides esparcidos.

Distribución. México, Nicaragua, Costa Rica, Antillas, Puerto Rico, Trinidad, Colombia, Venezuela, Guiana, Suriname, Guiana Francesa, Ecuador, Perú, Uruguay, Brasil (Da Silva et al., 2015) y extremo NE de Argentina (Fig.1).

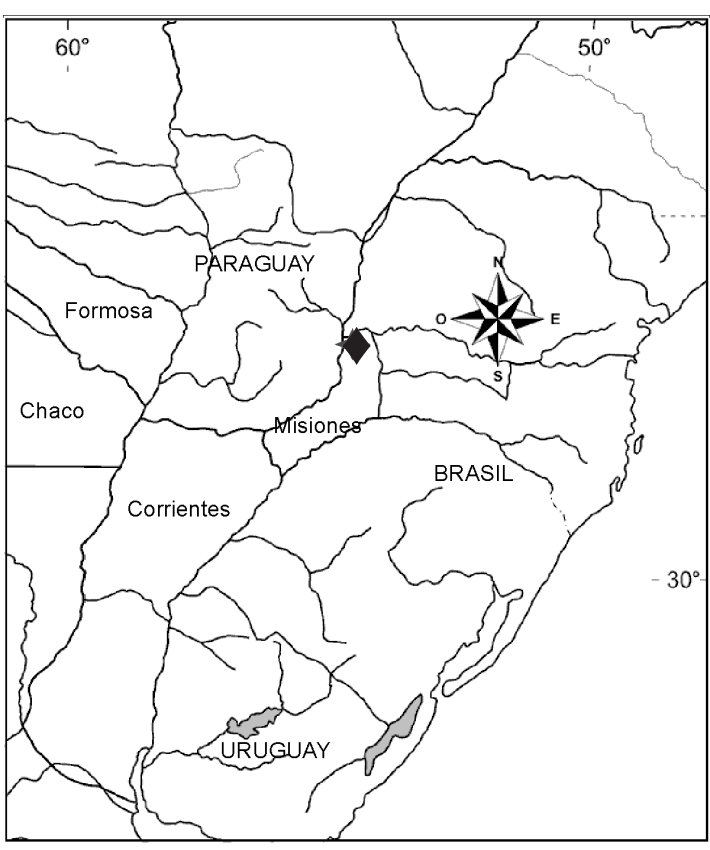

Fig. 1. Mapa donde se indica el hallazgo de la nueva cita con un rombo

Material examinado. ARGENTINA. Prov. Misiones: Depto. Iguazú, Pto. Bossetti, 25 48' 53,8" S - 54 37' 09,8" O, 06-IX-2017, Keller \& Rojas 13637 (CTES).

Observaciones ecológicas. el ambiente donde se halló la especie es un bañado de 4,5 has, con cobertura de árboles bajos, siendo las especie del dosel más abundante Copaifera langsdorfii Desf. (Fabaceae) con 390 ejemplares de más de $5 \mathrm{~cm}$ de diámetro por hectárea. Los sectores con mayor cobertura del sotobosque se constituyen de helechos y latifoliadas, mientras que en los espacios más abiertos predominan gramíneas y ciperáceas. Actinostachys pennula se oculta bajo el denso pastizal, donde comparte su hábitat con briófitas y diversas angiospermas de tamaño reducido, incluyendo micoheterótrofas.

Cabe destacar que el hallazgo de esta especie en el territorio misionero no es inesperado, ya que la misma se encuentra presente tanto en el sur de Brasil como en el este de Paraguay.

Observaciones taxonómicas. en relación al sinónimo controversial Actinostachys germanii 
Gonzalo J. Marquez y Héctor A. Keller - Actinostachys en Argentina


Fig. 2. Esporofito de Actinostachys pennula. A: Aspecto general. B: Detalle del rizoma piloso. C: Circino. D: Fronde fértil con esporangióforos inmaduros. E: Fronde fértil con esporangióforos maduros. Escalas en $\mathrm{cm}$ : A: 3; B: 1 ; C: 0,4; D: 0,8; E: 0,6 
Bol. Soc. Argent. Bot. 53 (3) 2018
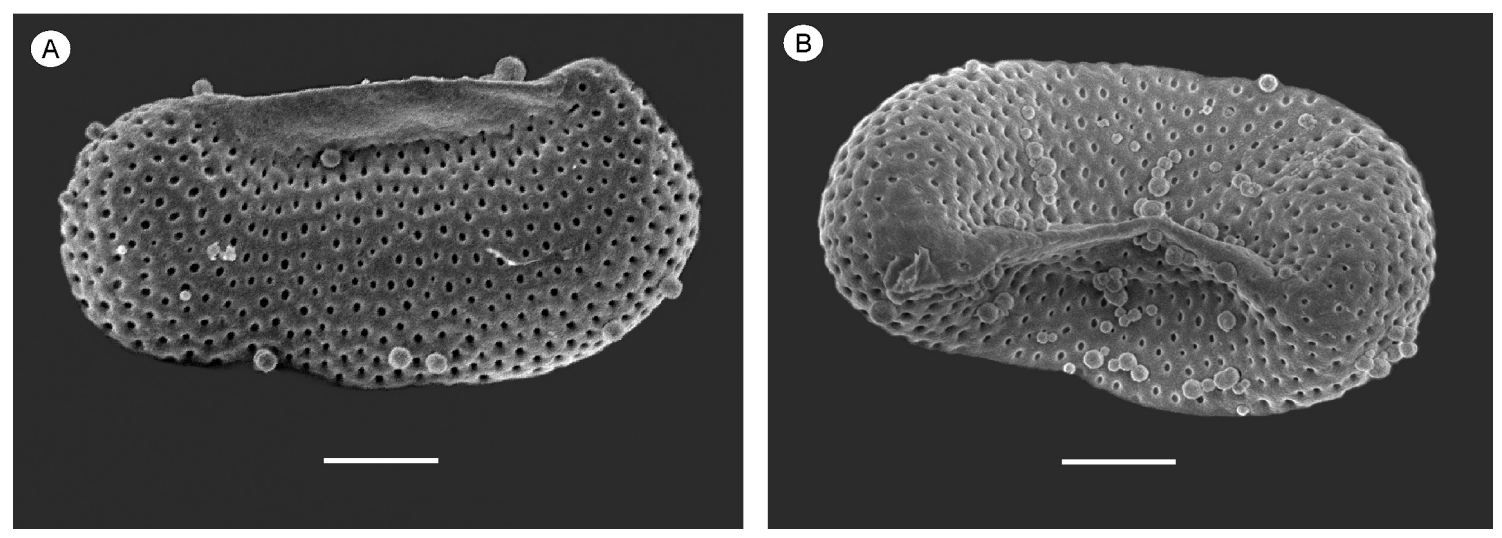

Fig. 3. Esporas foveadas de Actinostachys pennula, con esférulas en la superficie. A: Vista ecuatorial. B: Vista proximal. Escala: $10 \mu \mathrm{m}$.

Fée, y en base a los estudios realizados por Wagner \& Quevedo (1985) y Nauman (1987), quienes señalan que esta especie es una forma juvenil de $A$. pennula, consideramos a la primer especie sinónimo de la segunda.

\section{Agradecimientos}

Los autores agradecen a la Ing. Liliana Rivero (Arauco S.A.) y al Lic. Gustavo Aparicio (Fundación Hábitat y Desarrollo) por posibilitar la campaña de recolección en Pto. Bossetti. A José L. Rojas por su colaboración en campo.

El presente trabajo fue financiado por el subsidio PIP 0169 (CONICET).

\section{Bibliografía}

BIERHORST, D. W. 1971. Morphology and anatomy of new species of Schizaea and Actinostachys. Amer. J. Bot. 58: 634-648.

DA SILVA, F. D., F. P. ATHAYDE FILHO \& P. G. WINDISCH. 2015. Schizaeaceae (Polypodiopsida) no Estado do Mato Grosso, Brasil. Pesquisas Bot. 68: 107-118.

KELLER, H. A. 2017. Aenigma, un género sudamericano de Apocynaceae (Asclepiadoideae) con tricomas vibrátiles en la corola. Lilloa 54: 58-73.
KELLER, H. A. \& S. LIEDE-SCHUMANN. 2017. "The end of an enigma", a new subtribe and nomenclatural novelties in Asclepiadeae (Apocynaceae: Asclepiadoideae). Bonplandia 26: 133-136.

KRAPOVICKAS, A. 2014. Nuevas especies de Sida, sección Sida (Malvaceae). Bonplandia 23: 65-118.

MEZA TORRES, E., S. FERRUCCI, H. A. KELLER \& G. MARQUEZ. 2006. Presencia de Doryopteris lomaricea (Pteridaceae-Pteridophyta) en Argentina. Bonplandia 15: 143-148.

NAUMAN, C. E. 1987. Schizaeaceae in Florida. Sida 12: 69-74.

PPG I. 2016. A community-derived classification for extant lycophytes and ferns. J. Syst. Evol. 54: $563-$ 603.

PRADO, J. 2005. Flora da Reserva Ducke, Amazônia, Brasil: Pteridophyta-Schizaeaceae. Rodriguésia 56 (86): 93-97.

RAMOS GIACOSA J. P. \& M. C. BARAKAT. 2018. Spore morphology and wall ultrastructure of Actinostachys pennula (Sw.) Hook. and $A$. subtrijuga (Mart.) C. Presl. (Schizaeaceae). Palynology DOI:10.1080/01916122.2017.1413688

SHEN, H., D. JIN, J. SHU, X. ZHOU, M. LEI, R. WEI, H. SHANG, H. WEI, R. ZHANG, L. LIU, Y. GU, X. ZHANG \& Y. YAN. 2018. Largescale phylogenomic analysis resolves a backbone phylogeny in ferns. GigaScience 7: 1-11.

SMITH, A. R., K. M PRYER, E. SCHUETTPELZ, P. KORALL, H. SCHNEIDER \& P. G. WOLF. 2006. A classification for extant ferns. Taxon 55: 705-731. 
TRYON, A. \& B. LUGARDON. 1991. Spores of the Pteridophyta. Surface, wall structure and diversity based on electron microscope studies. Springer-Verlag, New York.

WAGNER, W. \& V. QUEVEDO. 1985. Polymorphism in Actinostachys pennula Sw. and the taxonomic status of Actinostachys germanii (Fée) Prantl. Amer. J. Bot. 72: 927-928.
YAÑEZ, A. \& M. PONCE. 2017. La familia Lindsaeaceae en Argentina. Bol. Soc. Argent. Bot. 52: 587-596.

Recibido el 9 de marzo de 2018, aceptado el 31 de julio de 2018. Editora: Olga Gladys Martínez. 
